\title{
POLYNOMIAL RINGS OVER GOLDIE RINGS ARE OFTEN GOLDIE
}

\author{
VICTOR CAMILLO AND ROBERT GURALNICK
}

\begin{abstract}
Here, we prove a result that has as a consequence the fact that if the ring $R$ is an algebra over an uncountable field the a.c.c. on annihilators is preserved under polynomial extensions by any number of variables.
\end{abstract}

Recently Jeanne Kerr [1] has given an example of a ring $R$ with ascending chain condition on annihilators that has the property that the polynomial ring $R[X]$ does not have the ascending chain condition on annihilators (a.c.c.). This answered a questin of long standing duration on the behavior of the classical Goldie conditions under polynomial extensions. Kerr's example is an algebra over $Z_{2}$.

LEMMA. Let $R$ be a ring which contains an uncountable set $V$ in the center of $R$ having the property that if $u, v \in V$ and $u \neq v$, then $u-v$ is a nonzero divisor. Let $S$ be a countable subring of $R$. Then, there is an infinite subset $V^{\prime}$ of $V$ such that $V^{\prime}$ is algebraically independent over $S$.

ProOF. Let $V^{\prime}$ be a maximal subset of $V$ which is algebraically independent over $S$. Suppose $V^{\prime}$ is finite. Then, any $v \in V$ satisfies a nonzero polynomial in the ring $\left(S\left[V^{\prime}\right]\right)[X]$. Since there are uncountably many elements in $V$ and only countably many polynomials, there is a polynomial $f(x)$ with infinitely many roots in $V$. Let $u$ and $v$ be two of these. Since we may divide by monics in any ring, $f(x)=(x-u) h(x)$, but $f(v)=0=(v-u) h(v)$ and $v-u$ is regular so $h(v)=0$, and continuing, we obtain a contradiction since the number of roots is larger than the degree of $f(x)$.

THEOREM. Let $R$ be a ring containing an uncountable set $V$ in the center of $R$, so that if $u$ and $v$ are distinct elements of $V, u-v$ is a nonzero divisor. Let $P$ be a property so that

(*) $\quad A$ ring $T$ satisfies $P$ if and only if every countable subring of $T$ satisfies $P$ (e.g., the a.c.c. on annihilators).

Then $R$ satisfies $P$ if and only if $R[X]$ satisfies $P$, where $X$ is any set of variables.

ProOF. One way is trivial. Conversely, we show $R[X]$ satisfies $P$ by showing that every countable subring $R_{0}$ of $R[X]$ does. Clearly, $R_{0} \subset S\left[x_{1}, x_{2}, \ldots\right]$ for some countable subring $S$ of $R$ and countable set of variables. Choose $V^{\prime}$ as in the lemma so that $S\left[x_{1}, x_{2}, \ldots\right] \approx S\left[V^{\prime}\right] \subset R$. Then, since $R_{0} \subset S\left[x_{1}, x_{2}, \ldots\right], R_{0}$ has $P$, so that by $* R[X]$ does.

Received by the editors November 11, 1985.

1980 Mathematics Subject Classification (1985 Revision). Primary 16A34, 16A33; Secondary 16A99. 
COROLLARY. If $R$ is an algebra over an uncountable field and $X$ is any set of variables then $R$ has the ascending chain condition on annihilators if and only if $R[X]$ does.

REMARK. The natural proof of the above, say in one variable, consists in the following: Any statement about chains of annihilators involves only countably many polynomials. Now, find an element in the field on which none of these vanish and embed the problem in $R$ by evaluation at this element.

Schock [2] proved that finite dimensionality is preserved by polynomial extensions, so that

COROLLARY. If $R$ is an algebra over an uncountable field, $R$ is Goldie if and only if $R[X]$ is Goldie.

\section{REFERENCES}

1. Jeanne Kerr, (to appear).

2. Robert Schock, Polynomial rings over finite dimensional rings, Pacific J. Math. 42 (1972), 251257.

DEPARTMENT OF MATHEMATICS, UNIVERSiTy OF IOWA, IOWA City, IOWA 52242

Department of MATHEMATiCs, UNiversity of SOUTHERn CAlifornia, Los ANGELES, CALIFORNIA 90089 